\title{
ALLEVIATE EXPOSED NODE ISSUES IN WIRELESS MESH NETWORK (WMN) USING A NOVEL APPROACH OF CONCURRENT MEDIUM ACCESS CONTROL (C-MAC) PROTOCOL
}

\author{
Vigneswara Rao Gannapathy ${ }^{1}$, Ahamed Fayeez Bin Tuani Ibrahim ${ }^{2}$, Zahriladha Bin Zakaria ${ }^{3}$, Abdul \\ Rani Bin Othman ${ }^{4}$, Mohamad Kadim Bin Suaidi ${ }^{5}$ \\ ${ }^{1}$ Lecturer, Department of Electronics and Computer Engineering, Universiti Teknikal Malaysia Melaka, Melaka \\ (UTeM), Malaysia \\ ${ }^{2}$ Lecturer, Department of Electronics and Computer Engineering, Universiti Teknikal Malaysia Melaka, Melaka \\ (UTeM), Malaysia \\ ${ }^{3}$ Lecturer, Department of Electronics and Computer Engineering, Universiti Teknikal Malaysia Melaka, Melaka \\ (UTeM), Malaysia \\ ${ }^{4}$ Lecturer, Department of Electronics and Computer Engineering, Universiti Teknikal Malaysia Melaka, Melaka \\ (UTeM), Malaysia \\ ${ }^{5}$ Lecturer, Department of Electronics and Computer Engineering, Universiti Teknikal Malaysia Melaka, Melaka \\ (UTeM), Malaysia
}

\begin{abstract}
The importance and high growth of the communication infrastructure is clearly stated under the Malaysia Economic Transformation Programme (ETP). The most popular technology that used to provide the internet service to public is the IEEE $802.11 \mathrm{a} / \mathrm{b} / \mathrm{g} / \mathrm{n}$ based WiFi. However, the coverage provided by the WiFi technology is only limited up to certain range. In addition, the performance of the WiFi network also will become poorer and poorer as the number of users associate with that WiFi network increases. In order to address these issues, the best topology configuration known as mesh is proposed by wireless research community. This technology is also called as Wireless Mesh Networks (WMN) and it became an emerging technology which is plays an important role in the next generation wireless communication. However, the implementation of IEEE 802.11 Medium Access Control (MAC) protocol in WMN significantly degrades the network performance due to the presence of exposed node problem. The presence of exposed nodes in WMN caused waiting delay (prevent the surrounding neighboring nodes to involve in transmission activity for entire duration of ongoing transmission) and it is subsequently leads to poor throughput achievement. The significance of this research is to investigate the cause of exposed nodes that degrade the performance of WMN and proposed a novel protocol to solve it to provide better network performance and throughput.
\end{abstract}

Keywords: Wireless Mesh Network (WMN), Medium Access Control (MAC), Exposed Node, and Concurrent Transmission

\section{INTRODUCTION}

The establishment of access rights proposed in IEEE 802.11 MAC (IEEE, 1998) is far more difficult in multihop WMN. It is observed by many researchers that the throughput of the IEEE 802.11 MAC protocol drop when it is applied directly in multihop networks such as WMN [1, 2]. The IEEE 802.11 MAC protocols face the problems of exposed node when implemented in multihop wireless network. The IEEE 802.11 MAC protocol, tackle the issues of hidden node problem by employing a four way handshake of RTS-CTSDATA-ACK packets.

According to the handshake mechanism, the source node will send the RTS and DATA packets while the destination node will respond with CTS and ACK packets. All the nodes which are in the range either source or the destination nodes have to remain silent and refrain from transmit any packet until entire transmission is completed. This virtual carrier sensing mechanism avoids the collision of DATA packet at recipient node and CTS or ACK packets at sender node. Even though this mechanism alleviates the hidden node problem yet the exposed node problem still remain unsolved.

The IEEE 802.11 MAC protocol effectively precludes other neighboring nodes from involving in transmission activity during the entire duration of ongoing packet transmission. This will introduced the problem called exposed node thus degrades the performance accordingly. These nodes will remain silent and refrain from initiate the transmission for the duration as specified in their NAV. In addition, when the number of competing nodes increases in the network, thus the number of exposed node also increases accordingly. 
The exposed node problem can be elaborated more by referring to Figure 1. After proper exchanged of RTS/CTS control packets between node R1 and A, they will begins their DATA transmission. During this entire packet transmission, node $\mathrm{B}$ does not allow to receive the packet from any of its neighboring nodes since it already silenced by CTS control packet from node A (i.e. virtual carrier sensing Mechanism). This is known as exposed node problem. An efficient MAC protocol could allow node B to receive the DATA packet from R2 since it doesn't conflict with the ongoing transmission from node R1 to node A.

Figure 2 shows different scenario of exposed node problem. After proper exchanged of RTS/CTS control packets between node $\mathrm{A}$ and R1, they will begins their DATA transmission accordingly. During this entire packet transmission, node $\mathrm{B}$ does not allow to involve in transmission activity with any of its neighboring nodes since it already silenced by RTS control packet from node A (i.e. virtual carrier sensing Mechanism). This is also known as exposed node problem. An efficient MAC protocol could allow node $\mathrm{B}$ to transmit the DATA packet to R2 concurrently since it doesn't conflict with the ongoing transmission from node A to node R1.

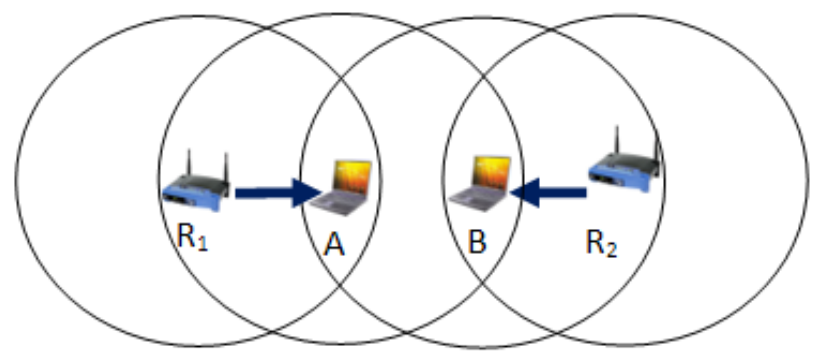

Fig -1: Exposed Node Problem Cause by Virtual Carrier Sensing (Scenario A)

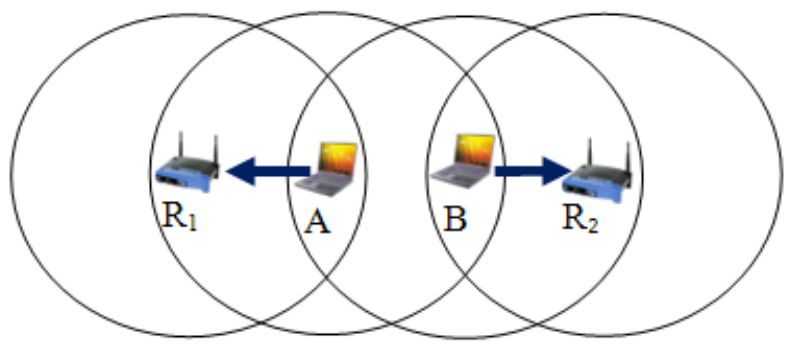

Fig -2: Exposed Node Problem Cause by Virtual Carrier Sensing (Scenario B)

Thus, under the IEEE 802.11 DCF MAC protocol, it effectively precludes other one hop neighboring nodes from involve in transmission activity during the entire duration of ongoing packet transmission and cause exposed node problem thus degrades the network performance.

Due to these reasons, the throughput of WMN degrades significantly thus a good MAC protocol shall design to boost the performance. Therefore, to suit and support the multihop WMN, several MAC protocols have been developed by the researchers. The main aim of the developed protocols is to improve the overall throughput of a multihop WMN. The following section will discuss some related works that had been proposed by researchers in order to solve the exposed node problem by enabling the concurrent transmission.

\subsection{Concurrent Transmission Access Protocols}

The concurrent transmission approaches have been proposed by many researchers as a transmission strategy to combat exposed node problems which is common in IEEE 802.11 MAC protocol. Its restrictive nature to disallow other neighboring nodes from involves in the transmission activity until the ongoing transmission complete degrade the throughput of WMN significantly. The throughput can be improved by using the concurrent transmission approaches. Thus, it can achieve better channel utilization and spatial reuse. By enabling the concurrency, it boosts its throughput and also alleviates the exposed node problem. The following section will discuss some related approaches that had been proposed to solve exposed node problem by enabling concurrent transmission approach thus gain better channel utilization.

\subsection{Opportunistic Approach in Mitigating Exposed}

\section{Node Problem}

One interesting work has proposed in [3] to mitigate the expose node problem. The nodes implemented with this protocol will recognize themselves as an exposed node by hearing the basic sequence of packets (i.e. the RTS control packet followed by the DATA packet from the same node) and opportunistically schedule concurrent transmission whenever possible. For example, when the exposed nodes (i.e. E1, E2 and E3) in the Figure 3 overhear the basic sequence of packets, it will align their DATA packet transmission with ongoing transmission. This communication does not require any additional RTS/CTS exchange. All exposed nodes (known as secondary transmission in this work) will tries to squeeze its transmission with primary node when it's DATA transmission is in progress. This approach effectively preclude from schedule secondary transmission, when multiple transmissions are in progress.

However the protocol enables the concurrency by the exposed node which is caused by the NAV RTS only. A part from that, the packet size of the primary transmission must be larger than the packet size of secondary transmission. This is another constraint that was highlighted by the author in this work to enable the concurrent transmission successfully. Since the packet size of primary transmission is larger than the secondary transmission, thus the secondary transmission is required to defer for some amount of time before finishing its transmission. This to make sure both primary and secondary transmission can finish exactly at the same time before commencing the ACK transmission. This deferral is to avoid the collision between DATA and ACK packets. 


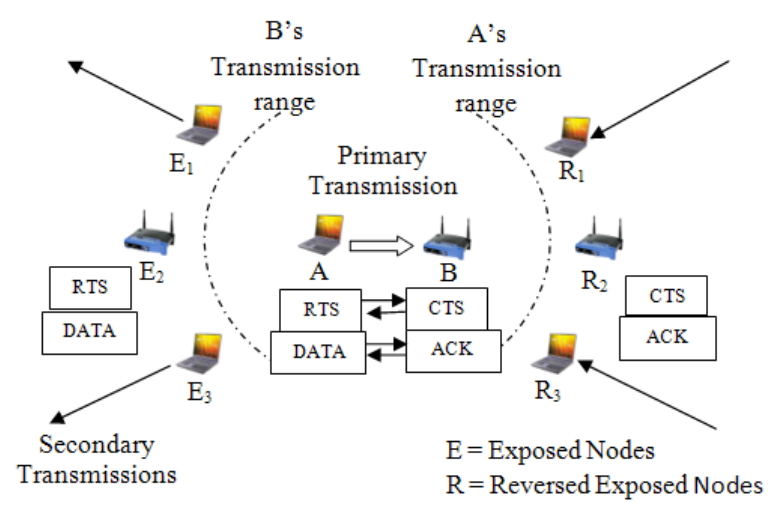

Fig -3: Opportunistic Approach in Mitigating Exposed Node Problem

\subsection{Multiple Access Collision Avoidance with Parallel Transmission (MACA-P)}

The earliest work on enabling concurrent transmission in WMN is Cooperative Medium Access Scheme [4] that contributes to significant improvement in the overall throughput of WMN. This protocol is improved version of $[5,6]$, with the introduction of Multiple Access Collision Avoidance-Parallel or MACA-P protocol. Figure 6 shows the pattern of the concurrent transmission that could be allowed in MACA-P without the collision.

This mechanism improves the utilization of channel by adopting spatial-reuse technique. A 'control gap' is introduced between the exchange of RTS/CTS control packets and subsequent exchange of DATA/ACK packets. The control gapwill begin at first sender - receiver pair of communicating node (i.e. master node). All other neighbouring nodes will be exploited by RTS from master node to complete their own RTS/CTS within this control gap and to align their DATA/ACK transmission with the DATA and ACK phases of master node.

In other word, this control gap is introduced to synchronize the DATA and ACK period of all concurrent transmission to avoid them colliding between each other (i.e. DATA packet of a transmission and ACK packet of another transmission). To achieve this DATA and ACK alignment, the RTS and CTS control packets were designed to carry the start time of DATA and ACK transmission. The detail refinements of MACA-P are described in [4, 5, and 6].

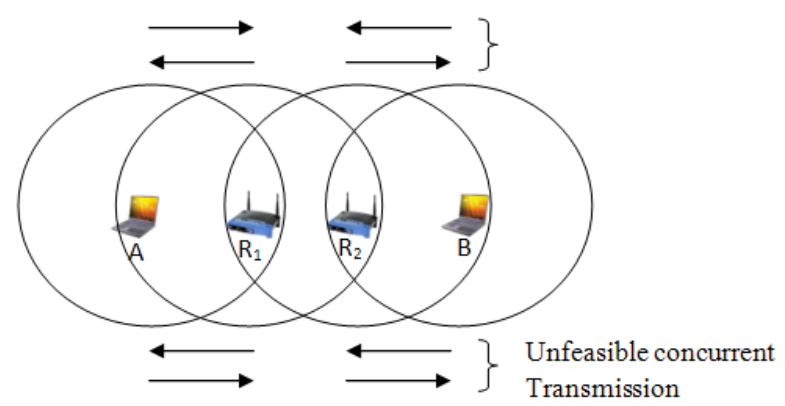

Fig -4: Feasible and Unfeasible Concurrent Transmission Pattern
However MACA-P is only performing well for specific scenario when large size of payload is considered. In addition, this protocol also caused wasted deferral time due to infeasible scheduling of concurrent transmission especially when low traffic is considered at intermediate nodes in multihop WMN communication.

\section{CONCURRENT MEDIUM ACCESS CONTROL (C-MAC) PROTOCOL}

Exposed node is not really serious problem in low-density wireless mesh network but it becomes critical in highdensity environment. The future wireless mesh networks must capable to accommodate high-density user with low delay and high throughput. The first problem to be tackled is how to allow multiple transmissions to happen. The answer is either we use multiple channel or/and multiple radio solution or by modifying the existing MAC protocol to allow concurrent transmission. The former is known to be more expensive than the latter due to hardware modification that we try to avoid, which leaves us with the latter solution. In this work, we propose modification at the control phase part (includes RTS-CTS) to allow concurrent transmission. The Basic Structure of C-MAC

The proposed protocol, namely, Concurrent Medium Access Control (C-MAC) protocol enables concurrent transmission to solve the exposed node issues. In order to smoothen the concurrent operation, some enhancements has made in control packets (RTS, CTS and ACK) as per described below:-

1. Request-to-Send (RTS): The RTS control packet is used for initiating a DATA packet transmission. It was modified to hold the frame control field, MAC addresses of the transmitter and receiver, $T_{\text {DATASTR }}$ and $\mathrm{T}_{\mathrm{ACKSTR}}$ field, Route Identification $\left(\mathrm{R}_{\mathrm{ID}}\right)$ field and also FCS field. The modified RTS control packet known as $m$ RTS. The size of the $m$ RTS control packet is 26 Bytes.

2. Clear-to-Send (CTS): The CTS control packet will be responded by the receiver node to indicate it readiness to receive the DATA packet. It was modified to hold the frame control field, MAC addresses of the transmitter and receiver, $\mathrm{T}_{\text {DATASTR }}$ and $\mathrm{T}_{\mathrm{ACKSTR}}$ field, Route Identification $\left(\mathrm{R}_{\mathrm{ID}}\right)$ field, SS-bit field and also FCS field. The modified CTS control packet known as $m$ CTS. The size of the $m$ CTS control packet is 26 Bytes.

3. Data (DATA): The data message contains the actual data and includes all the higher layer protocol header and payload.

4. Acknowledgement (ACK): The ACK control packet is used by the receiver node to acknowledge back the correct reception of the DATA packet to sender node. It is used as a mechanism to trigger fast retransmission of collided DATA packet. The modified ACK control packet known as $m$ ACK. The size of the $m \mathrm{ACK}$ control packet is 18 Bytes. 
In the scenario (see Figure 5), let's assume that both client A and $\mathrm{B}$ compete among each other to get the access to channel. The first pair communicating node, known as master node which is considered as a node who manage to get the access to channel for initiation process. In the given scenarios, let's assume client A manages to grab the channel and initiates its transmission according to four-way handshakes mechanism. The transmission between client A to router $R_{1}$ is considered as the first pair communicating node (master node). The main task of master node is to establish the exchange phase and inform all other one hop neighboring nodes to exchange their respective $m \mathrm{RTS} / m \mathrm{CTS}$ control packets within remaining portion of exchange phase and to schedule their DATA $/ m$ ACK with master node (as shown in Figure 5). The master node (i.e. client A), will schedule the DATA transmission at the end of exchange phase and schedule the $m \mathrm{ACK}$ transmission at the end of DATA transmission phase.

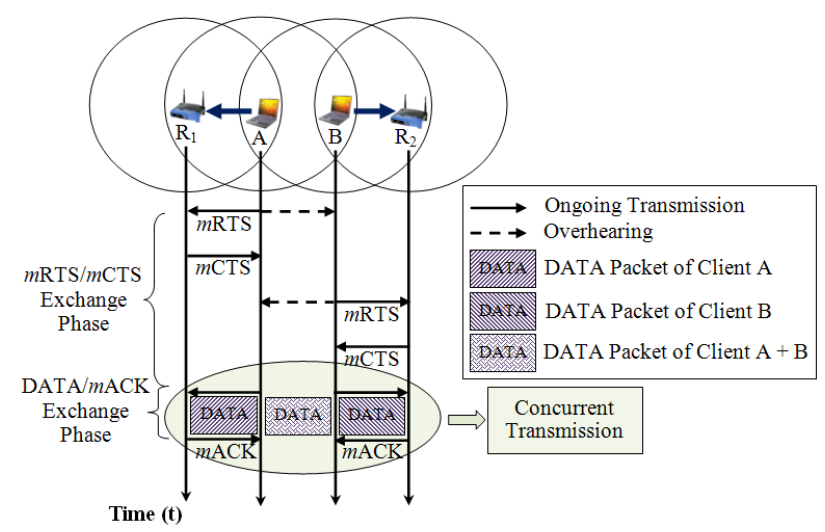

Fig -5: Scheduled Concurrent Transmissions

The master node (i.e. client A), will launch the exchange phase through its $m$ RTS control packet and invites all other possible one hop neighborhood nodes who overhear this $m$ RTS control packet to schedule their $m$ RTS $/ m$ CTS exchange within this exchange phase gap. Thus, this exchange phase will be utilized by second pair of communicating node (i.e. client B), known as slave node, to schedule its transmission with master node. The $m \mathrm{RTS} / m \mathrm{CTS}$ exchange of the slave node will be happened within this exchange phase gap thus this approach intelligently solved the collision happens due to role reversals.

Upon completion of exchange phases, both nodes (master and slaves) will wait for the DATA and mACK packet transmission phase. At this point both DATA packet transmission from client $A$ to router $\mathrm{R}_{1}$ and from client $\mathrm{B}$ to router $R_{2}$ will be occurred concurrently. It is noted that if there was no $m \mathrm{ACK}$ phase introduced in the protocol, two nodes able to transmit concurrently their DATA without worrying about the DATA and $m$ ACK transmission phases. Furthermore, the exposed node problem can be solved partially. However, the $m \mathrm{ACK}$ packet is the only way of node able to know on the successfully or failure of a transmitted DATA packet. Thus, to increase the reliability of the DATA packet, the mACK packet will be sent out by both destination nodes (i.e. router $R_{1}$ and router $R_{2}$ ) concurrently upon completion of first pair DATA packet transmission. Therefore, the size of DATA packet of first pair must be larger than the size of DATA packet of second pair. This scenario can be clearly elaborated by referring to the timing diagram as shown in Figure 6.

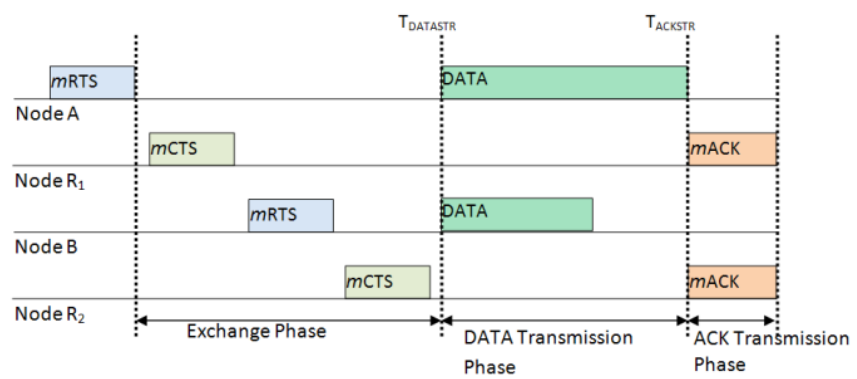

Fig -6: Timing Diagrams of Scheduled Concurrent Transmissions

\section{RESULTS}

In this session, the performance parameters, namely theoretical throughput was analyzed as a function of number of competing node. Analysis was conducted to analyze the end-to-end delay and throughput when the number of the competing nodes increases accordingly. The effect of throughput was analyzed by varying the number of competing nodes from 1 to N. Figure 7 depicts the variation throughput as a function of number of competing node for the various MAC protocols such as IEEE 802.11 MAC, MACA-P and MARCH. A node among all competing nodes [ $\mathrm{N}-(\mathrm{N}-1)]$, who manage grabs the channel to initiates the transmission at first is considered as master node while all other nodes $(\mathrm{N}-1)$ who squeezes and schedule their transmission (to enable concurrent transmission) with master node is considered as slave nodes.

According to IEEE 802.11 MAC protocol, the master node will immediately initiate its transmission without further delay because it is not affected by exposed node problem. Meanwhile, the slaves' nodes have to delay their transmission for certain amount of time because they are exposed to other ongoing transmission. The worst case of the node is defined as starving node in which it has to wait for all other competing nodes to complete their transmission before it commences into the transmission. The transmission of worst case node can only take place upon completion transmission of node $1,2, \ldots(\mathrm{N}-1)$. The size of exchange phase that proposed by the master node is assumed varied accordingly in order to enable all the slave nodes to schedule concurrent transmission and the time is not wasted in idling.

The packet size of slave nodes is set to 2048 bytes and it smaller compare to the packet size of master node which is set to 4096 bytes. In order to enable the efficient concurrency, the DATA packet size of the master node must be larger than the DATA packet size of slave. 
It is obvious from the plot in the Figure 8 that when the number of competing node is varied in the range of 2 to $\mathrm{N}$ nodes, C-MAC protocol shows the most efficient delay consumption for compare to the 802.11 MAC protocol. This is due to its capability of concurrent transmission. The performance was evaluated in term of theoretical throughput.

The throughput achieved by C-MAC when it handles 2 concurrencies is 5.31 Mbps compare to other protocols which is only achieved 4.87 Mbps by MARCH, 2.83 Mbps by MACA-P and 2.19 Mbps by 802.11 MAC. Since the delay consumption seems small when implementing $\mathrm{C}$ MAC protocol especially when more number of competing nodes is considered, hence it gains better throughput. The throughput achieved by C-MAC when it handles 10 concurrencies is $3.89 \mathrm{Mbps}$ compared to other protocols which is only achieved $2.43 \mathrm{Mbps}$ by MARCH, $1.32 \mathrm{Mbps}$ by MACA-P and $0.59 \mathrm{Mbps}$ by $802.11 \mathrm{MAC}$. It is clearly visible from both the graphs in Figure 7 that C-MAC protocol gains better performance in term of end-to-end delay and throughput.

For such dense network scenarios, the end-to-end delay consumption seems smaller when implements C-MAC and hence provide greater throughput. This behavior is caused by concurrency characteristic that $\mathrm{C}-\mathrm{MAC}$ has in reduces the overheads. Obviously this observation shows that the $\mathrm{C}$ MAC reducing the latency caused by the exposed node phenomenon significantly.

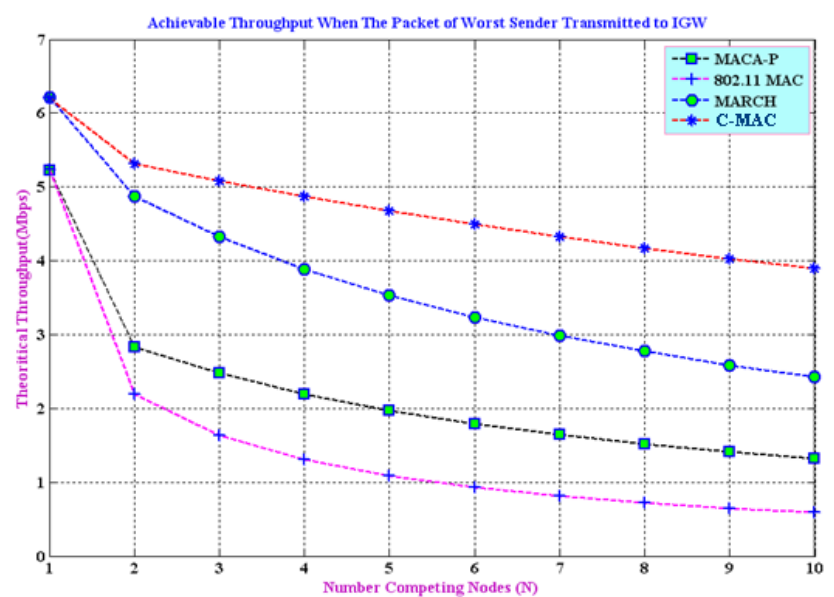

Fig -7: Achievable Throughputs When The Packet of Worst Node Transmitted to IGW

\section{CONCLUSION}

Multihop WMN plays an important role in the nextgeneration wireless communication. It promised a solution to provide ubiquitous wireless access at low cost and with architecture that easy to be deployed and maintained. A big challenge in designing WMN is to utilize the shared medium, the wireless communication channel, effectively. In this work, the issues and problems encountered by WMN were explored and studied in detail. In WMN, lack of concurrent transmission led to the exposed node problems thus caused ineffective channel utilization and subsequently led to throughput degradation. A novel protocol namely, Concurrent Medium Access Control (C-MAC) protocol was proposed in this work to encounter the exposed node problem in WMN. The performance of the proposed CMAC protocol was examined and compared with other three different collision avoidance MAC protocols such as MACA-P, MARCH and IEEE 802.11 MAC. Based on our analysis, the proposed C-MAC protocol seems to be more competent compare to other compared protocols in term of throughput and channel utilization. The result shows that the C-MAC protocol improves the network performance and turns to fulfill the requirement multihop WMN.

\section{ACKNOWLEDGEMENTS}

The authors would like to take this opportunity to thanks those who are contributes directly or indirectly in completion of this article and also for their constructive comments. In addition, the authors also would like to express our gratitude to Universiti Teknikal Malaysia Melaka (UTeM) for the support and encouragement. The work in this paper was funded by Exploratory Research Grant Scheme (ERGS) that offered to UTeM by Ministry of Higher Education, Malaysia.

\section{REFERENCES}

[1] R. C. Carrano, L. C. S. Magalhães, D. C. M. Saade, and C. V. N. Albuquerque, "IEEE 802.11s multihop MAC: A tutorial," IEEE Commun. Surv. Tutorials, vol. 13, pp. 52-67, 2011.

[2] G. Tripathi, N. G. Bawane, and R. V. Dharaskar, "Gateways and performance of WMN," in ICETC 2010 - 2010 2nd International Conference on Education Technology and Computer, 2010, vol. 2

[3] D. S. D. Shukla, L. C.-W. L. Chandran-Wadia, and S. I. S. Iyer, "Mitigating the exposed node problem in IEEE 802.11 ad hoc networks," Proceedings. 12th Int. Conf. Comput. Commun. Networks (IEEE Cat. No.03EX712), 2003

A. Acharya, S. Ganu, and A. Misra, "DCMA: A label switching MAC for efficient packet forwarding in multihop wireless networks," IEEE J. Sel. Areas Commun., vol. 24, pp. 1995-2004, 2006

[4] Acharya, A. Misra, and S. Bansal, "MACA-P: a MAC for concurrent transmissions in multi-hop wireless networks," Proc. First IEEE Int. Conf. Pervasive Comput. Commun. 2003. (PerCom 2003)., 2003

[5] Acharya, A. Misra, and S. Bansal, "Design and Analysis of a Cooperative Medium Access Scheme for Wireless Mesh Networks," BROADNETS, 2004

[6] V. R. Gannapathy, M. Kadim, B. Haji, M. Syahrir, B. Johal, L. K. Chuan, N. Bin Ramlit, and H. Mohamadt, "A Smooth Forwarding Operation in Wireless Mesh Network," no. October, pp. 83-87, 2011.

[7] V. R. Gannapathy, M. R. Ahmad, M. K. Suaidi, M. S. Johal, and E. Dutkiewicz, "Concurrent MAC with short signaling for multi-hop wireless mesh networks," in 2009 International Conference on 
Ultra Modern Telecommunications and Workshops, 2009

[8] V. R. Gannapathy, A. Fayeez, B. Tuani, Z. Bin Zakaria, A. Rani, B. Othman, and A. A. Latiff, "An Enhancement Of RTS / CTS Control Handshake In Csma / Ca Based Mac Protocol For An Efficient Packet Delivery Over Multihop Wireless Mesh Network ( WMN )," Int. J. Res. Eng. Technol. IJRET, vol. 02, no. 10, pp. 604-608, 2013

\section{BIOGRAPHIES}

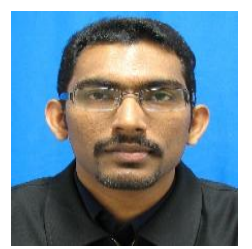

Engr. Vigneswara Rao Gannapathy currently serving as a lecturer in Universiti Teknikal Malaysia Melaka (UTeM) and he actively involves in research activities which is related to electronics and wireless networking. His research direction has focused on Wireless Mesh Networks which emerged as a key technology for next-generation wireless networking.

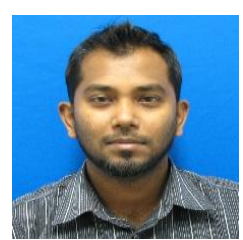

Ahamed Fayeez B Tuani Ibrahim, a Masters holder in Electronics \& Telecommunication, is a vivid computer networking enthusiast. His specific research interest includes low power network design and programming. He is a certified Cisco Network Analyst.

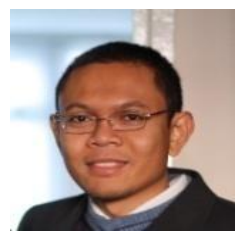

Dr. Zahriladha Zakaria, PhD, MIEEE, BEM, Grad IEM is currently working as a lecturer at the Faculty of Electronic and Computer Engineering, University Teknikal Malaysia Melaka. (UTeM). His research interests include a variety of microwave device development such as planar and nonplanar microwave filters, amplifiers and antennas.

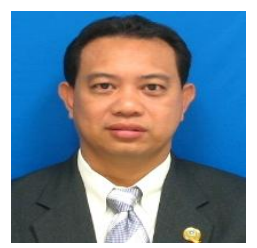

Associate Professor Dr. Abdul Rani Othman, $\mathrm{PhD}$, is currently working as lecturer at the Faculty of Electronic and Computer Engineering, University Teknikal Malaysia Melaka. (UTeM). His research interests include a variety of microwave device development, radio wave propagation, amplifiers and antennas.

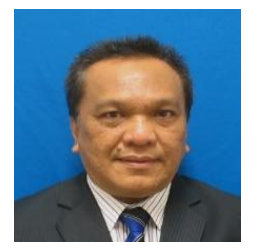

Professor Dato’ Dr. Mohamad Kadim Bin Suaidi, PhD, is currently Vice Chancellor (VC) of Universiti Malaysia Sarawak (UNIMAS). His research interests include Optoelectronics, Telecommunication, and Wireless Communication. He has a wide knowledge on high speed optical diagnostics of laser interactions and also on Microstrip Antennas and Arrays 